\title{
Right Eye
}

National Cancer Institute

\section{Source}

National Cancer Institute. Right Eye. NCI Thesaurus. Code C119333.

The eye positioned in the right orbit. 